\title{
CONOCIMIENTO TRADICIONAL SOBRE LAS PLANTAS MEDICINALES DE YARUMO (Cecropia sciadophylla), CARAMBOLO (Averrhoa carambola) Y UÑA DE GATO (Uncaria tomentosa) EN EL RESGUARDO INDÍGENA DE MACEDONIA, AMAZONAS ${ }^{1}$
}

\author{
Lina Paola Garzón Garzón ${ }^{2}$
}

Recibido el 28 de abril de 2015, aprobado el 25 de agosto de 2015 y actualizado el 24 de mayo de 2016

DOI: 10.17151/luaz.2016.43.17

\section{RESUMEN}

El conocimiento medicinal en el Resguardo Indígena de Macedonia se fundamenta en el manejo de las plantas medicinales, bajo la forma de "medicina casera", entre las cuales se destacan el Yarumo (Cecropia sciadophylla), el Carambolo (Averrhoa carambola) y la Uña de Gato (Uncaria tomentosa) por su valor de uso cultural. A través de entrevistas semiestructuradas aplicadas dentro de la comunidad, se pudo analizar aspectos relacionados con la incidencia de procesos migratorios en la adquisición de conocimiento, la transmisión de saberes sobre las plantas medicinales, el desconocimiento sobre su uso medicinal, el reporte de usos de estas plantas y las prácticas en la preparación de remedios. Se destaca que el conocimiento medicinal sobre estas plantas se encuentra altamente permeado por procesos de migración dentro del territorio amazónico y el transnacionalismo, el cual está concentrado en su mayoría por los abuelos sabedores, quienes han tenido más contacto y han experimentado con estas plantas. En este estudio se pudieron reportar 17 usos para la Uña de Gato, seguido de 11 para el Carambolo y 10 para el Yarumo.

\section{PALABRAS CLAVE}

Plantas medicinales, etnobotánica, medicina tradicional, resguardo indígena, Amazonas. 


\title{
TRADICIONAL KNOWLEDGE ABOUT MEDICINAL PLANTS OF YARUMO \\ (Cecropia sciadophylla), CARAMBOLO (Averrhoa carambola) AND CAT'S CLAW (Uncaria tomentosa) IN THE INDIGENOUS RESERVATION OF MACEDONIA, AMAZONAS
}

\begin{abstract}
Medicinal knowledge in the indigenous reservation of Macedonia is based on the management of medicinal plants such as "home remedies". Yarumo (Cecropia sciadophylla), Carambolo (Averrhoa carambola) and Cat's Claw (Uncaria tomentosa) can be stand out because their cultural use value. Using semistructured interviews on the community, it was analyzed aspects related to the impact of migration processes in the acquisition of knowledge, transmission of medicinal plants knowledge, ignorance of its medicinal use, report uses of medicinal plants and practices in the remedies preparation. The medicinal plants knowledge is highly permeate by migrational process on the Amazon territory and transnationalism. This was mostly concentrated in the grandparents knowing who had more contact and have experienced with the plants. This study reported 17 uses from Cat's Claw, followed by 11 uses of Carambolo and 10 uses of Yarumo.
\end{abstract}

\section{KEY WORDS}

Medicinal plants, ethnobotany, traditional medicine, indigenous reservation, Amazon.

\section{INTRODUCCIÓN}

La medicina tradicional es también considerada como un sistema complejo conformado por elementos como conocimientos, tradiciones, prácticas y creencias, los cuales se estructuran organizadamente a través de sus propios agentes conocedores (especialistas terapeutas, parteras, promotores, sobadores). Éstos poseen un método propio de diagnóstico y tratamiento; así como recursos terapéuticos propios, los cuales son reconocidos como las plantas medicinales (Organización Panamericana de la Salud, 2006). 
Más específicamente, la medicina tradicional indígena comprende aquellas prácticas médicas que han tenido su origen antes del periodo de la Colonia, en los espacios sociales y geográficos de las comunidades indígenas; así como un surgimiento en procesos donde la metodología terapéutica se basa en la historia, la cosmovisión y la identidad cultural indígena (Page, 1995). Lo anterior, hace énfasis en la característica de arraigo a una tradición cultural, permitiendo que se pueda hablar de distintas medicinas tradicionales de acuerdo a la comunidad y la zona geográfica en donde se practique (Zuluaga y Correa, 2002).

La estrecha relación salud-naturaleza se puede entender a través del ejercicio de la medicina tradicional de los sistemas de conocimiento chamánico, que involucra el saneamiento y la legalización territorial, su ordenamiento y aprovechamiento de los recursos. Al ser concertados estos aspectos en un comanejo de áreas protegidas y territorios indígenas, se evidencia también la relación salud-territorio (Zuluaga y Correa, 2002).

Asimismo, esta relación se materializa a través de las plantas medicinales que corresponden a especies vegetales con principios activos que tienen propiedades terapéuticas comprobadas empírica o científicamente (Organización Panamericana de la Salud, 2006). Éstas producen metabolitos secundarios útiles para la solución de problemas específicos de salud en el hombre; reflejándose así, el efecto de la naturaleza en la química y biología requerida para este proceso. Los médicos tradicionales tienen un amplio conocimiento de esta relación y de la importancia de estas plantas, no solo para el campo biomédico (diagnóstico, curación o prevención de enfermedades) sino también para el ordenamiento territorial y cultural de los grupos étnicos (Zuluaga, 1999).

Lo anterior, hace referencia al hecho de que muchas plantas medicinales hacen parte de la historia de la comunidad y sus dueños reales pueden ser espíritus que se encuentran en otro mundo (al cual solo pueden acceder los chamanes) o seres inmortales que nunca fueron humanos; por consiguiente, su uso va a depender de la negociación que haga el chamán con estos seres y del permiso que le sea otorgado (Quintana, 2009). Para Buitrago (2007) este uso de las plantas va más allá de lo práctico pues implica recordar y memorar sus historias, así como develar su origen. Debido a esto, la historia mítica de la planta es clave para su uso, pues da cuenta de las razones por las cuales son empleadas por la comunidad.

La concepción de medicina tradicional Tikuna3 de Macedonia, se enfoca en las actuaciones adecuadas o inadecuadas de la persona, ya que estas influyen 
directamente sobre su salud o enfermedad. De esta manera, la cura radica más en el cambio de los comportamientos que moralmente no son aceptados por la comunidad, que en el tratamiento físico del cuerpo (Quintana, 2009).

Con base en esta percepción, más allá de una dimensión biológica, se puede hablar de la relación de las enfermedades con la moral concebida en la comunidad que se justifica con la conformación de un cuerpo cultural (Pinzón y Suárez, 1992). No obstante, esta relación no es legitimada por la medicina tradicional, y por lo tanto, la forma de tratar la enfermedad no va a ser similar, pues no encaja con la validez del conocimiento dado por la racionalidad occidental (Sánchez, 1999).

En este Resguardo existe un manejo de las plantas medicinales, bajo la forma de "medicina casera", en donde se elimina el chamanismo, curanderismo u otra forma de rito pagano que vaya en contravía con la fe evangélica manejada en la comunidad (Barbosa, 2006). Dentro de las plantas medicinales con mayor uso cultural en Macedonia se encuentran el Yarumo (Cecropia sciadophylla), el Carambolo (Averrhoa carambola) y la Uña de Gato (Uncaria tomentosa), las cuales son empleadas por los indígenas a través de un conocimiento que está ligado a la relación de cada individuo con el medio físico, dada por sus vivencias (López, 2010).

El objetivo de este trabajo es hacer un análisis sobre el conocimiento tradicional en el Resguardo Indígena de Macedonia, a partir del uso de las plantas medicinales de Yarumo, Carambolo y Uña de Gato. Para esto, se tendrán en cuenta aspectos relacionados con la incidencia de procesos migratorios en la adquisición de conocimiento, la transmisión de saberes sobre las plantas medicinales, el desconocimiento sobre el uso medicinal de las plantas, el reporte de usos de estas plantas y las prácticas en la preparación de remedios.

\section{MATERIALES Y MÉTODO}

\section{Área de estudio}

El asentamiento Tikuna de Macedonia se encuentra ubicado en el Trapecio Amazónico a 50 km de Leticia (Figura 1), capital del departamento del Amazonas (Tobón y Ochoa, 2010). El Resguardo se localiza en la zona de amortiguación del Parque Nacional Natural Amacayacu y limita al norte con la comunidad de 
Mocagua, la quebrada Mata-matá y la finca El Zapote; al sur con la comunidad del Vergel; al oriente con los baldíos nacionales y al occidente con el río Amazonas (Parques Nacionales Naturales, 2005).

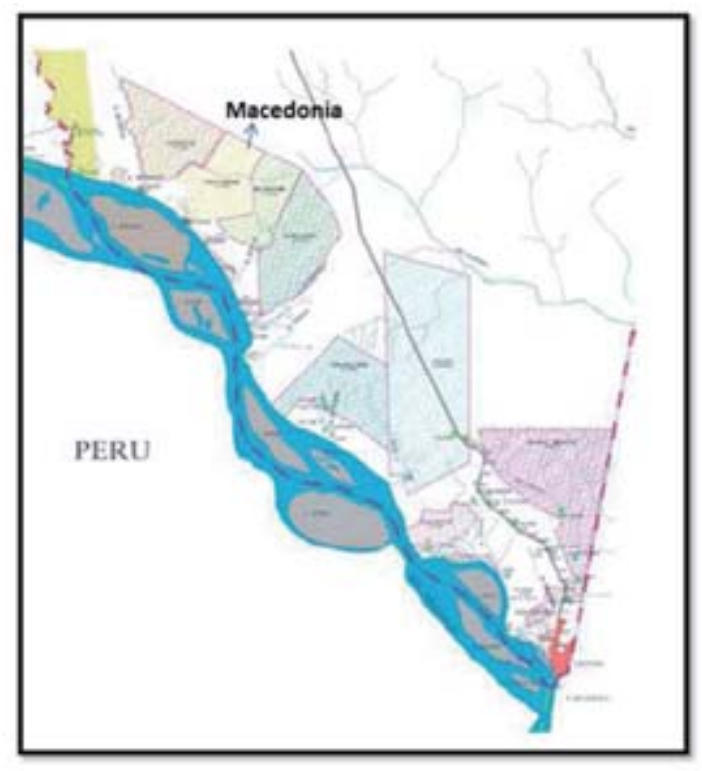

Fuente: Alcaldía de Leticia (2002).

Figura 1. Localización del Resguardo Indígena de Macedonia.

\section{Metodología}

Se realizaron entrevistas semiestructuradas a indígenas residentes de la comunidad, en forma aleatoria simple, los cuales se dividieron en tres grupos etarios: de 25 a 42 años, de 43 a 60 años y de 61 a 79 años. Estas personas llevan habitando la mayor parte de su vida en la comunidad; por consiguiente, pueden dar cuenta de las transformaciones en el territorio, culturales, religiosas y sociales, que inciden en el establecimiento del uso de las plantas de Carambolo, Yarumo y Uña de Gato dentro del Resguardo.

Con base en esta información, se establecieron categorías de análisis cualitativo que permiten un acercamiento a los factores que influyen en la percepción y manejo del conocimiento sobre las plantas medicinales, las cuales correspondieron a: incidencia de procesos migratorios, transmisión de saberes, uso medicinal y prácticas medicinales. 


\section{RESULTADOS Y DISCUSIÓN}

\section{Incidencia de procesos migratorios en la adquisición de conocimiento}

Macedonia ha sido el resultado de procesos migratorios que conformó una comunidad pluriétnica con indígenas Tikuna en su mayoría, Cocama, Yagua y mestizos (Barbosa, 2006). Este suceso fue permeado por la llegada de la Iglesia Panamericana en los años 60 , instaurando la creencia evangélica como pilar de la comunidad y que fue profesada inicialmente por sus fundadores (Buitrago, 2007).

De las personas entrevistadas, el $43 \%$ corresponden a indígenas Tikuna que se establecieron en la comunidad en los años 70 cuando se conformó Macedonia como un Resguardo. Dentro de estos, se encuentran los hijos de los fundadores: Anthero León, Inés León y Cornelio León. Otros indígenas pertenecen a familias antiguas que conformaron el primer caserío de la comunidad (Buitrago, 2007): Manuel Jordán, Ricaurte Careca, Alfonso Peña, Tesorio Peña, Lucio León y Ramón Victorino.

Adicionalmente, el $29 \%$ pertenecen a familias inmigrantes Tikuna y Cocama que arribaron ente la época de finales de los años 70 y comienzos de los años 90: Alfonso Panduro, Gustavo Suárez, Pablo Pérez, Jesús Rodríguez, Germán Peña e Israel Bastos. Estas personas llegaron provenientes en su mayoría del Putumayo, de comunidades aledañas a Macedonia, y de Bogotá. En especial, la población Cocama se cree que proviene de la región del Ucayali, en el Perú (Goulard, 2003) por lo que su conocimiento del uso y preparación de la medicina tradicional puede basarse en el de los primeros grupos indígenas de esta región (Pezo, 2006).

El 28\% restante de los entrevistados son nietos de fundadores o hijos de familias inmigrantes que han vivido toda o la mayor parte de su vida dentro de la comunidad: Delfín Bastos, Máximo León, Elda Peña, Job Peña, Gustavo Suárez Lucas, Jorge Eliécer Vásquez. La mayoría de ellos han tenido o tienen actualmente cargos de poder dentro de la comunidad como curacas o pastores.

El reconocimiento de cómo fue el proceso de asentamiento dentro de la comunidad permite determinar la forma como se ha adquirido el conocimiento medicinal, especialmente en los abuelos sabedores y la forma como se ha permeado por 
efectos culturales y religiosos dentro de la comunidad. Por ejemplo, en una entrevista el abuelo Alfonso Panduro comentó sobre la forma como conoció el Carambolo y su uso medicinal: "Cuando yo era joven andaba mucho a Brasil, Putumayo, Puerto Leguizamo y Puerto Asís. Allá los abuelos del Putumayo curaban, preparaban ese remedio, y como yo soy inteligente yo tenía que practicar" (com. per., 2013).

Debido a la ubicación de Macedonia en un punto de frontera con Perú y Brasil, los procesos de apropiación del territorio y conocimiento medicinal han estado permeados por un transnacionalismo (Luque, 2012, pp. 2-23). Con este proceso se han podido establecer redes sociales producto de las prácticas cotidianas, que inciden sobre el conocimiento y la forma de apropiación del mismo (Unigarro, 2011).

Lo anterior, se traduce en que parte del conocimiento que tienen los abuelos sabedores y otras personas de la comunidad proviene del intercambio con indígenas de otras comunidades del Perú y Brasil, al igual que con otras comunidades amazónicas colombianas. Pablo Pérez señaló que su conocimiento sobre el uso medicinal del Yarumo para la tensión alta y el colesterol lo aprendió de personas provenientes del Brasil: "A mí enseñó un brasilero, me dijo para hacer y lo hice. Otro brasilero que vino de por allá de Tabatinga dijo que con eso se mejoró. Entonces yo lo hice y me sané con eso" (com. per., 2013).

También, el uso del Yarumo para tratar el dolor de ojo parece provenir del conocimiento de indígenas peruanos. El abuelo Manuel Jordán relató cómo aprendió este remedio de un médico tradicional: "Había un señor que vino del Perú, Juan Sangama, le enseñó a mi papá y él a nosotros” (com. per., 2013).

No solo el saber medicinal tradicional es permeado por el transnacionalismo, sino también el conocimiento de la forma física de las plantas propias de la región amazónica como la Uña de Gato (Rengifo, 2007) y otras que fueron traídas a la región como el Carambolo (Tello, García y Vásquez, 2002). En una entrevista realizada al abuelo Lucio León comentó la forma como vio por primera vez el Carambolo y una forma de dispersión de las semillas:

La primera vez que yo lo miré fue más que todo en el Perú. Como la gente del Perú viene y la gente va al Perú, va trayendo semillas hasta que acá también se tiene. Cuando veo una semilla yo le voy trayendo, siempre la 
tengo en el bolsillo para poder sembrarlas, porque hay unas que no hay acá. (com. per., 2013)

Transmisión de saberes sobre las plantas medicinales dentro de la comunidad

El conocimiento tradicional indígena puede ser entendido como un sistema acumulativo de conocimientos no formales, resultado de la observación empírica y la transmisión oral de experiencia que pasan de una generación a otra (Dias de Avila-Pires, 2007). En este sentido, el conocimiento sobre el uso medicinal de las plantas de Yarumo, Carambolo y Uña de Gato dentro de la comunidad debería presentarse por la transmisión de los abuelos a los más jóvenes (Vargas, 2010). Se encontró en el grupo de las personas con mayor edad un predominio del conocimiento del uso de las plantas medicinales, especialmente sobre la Uña de Gato y el Yarumo (82\% para ambas plantas). Contrario a esto, en el grupo de personas jóvenes se evidenció que la mayoría no sabía los posibles usos medicinales de la Uña de Gato (67\%). En el grupo etario de 43-60 años todos los individuos tenían conocimientos sobre algún uso medicinal del Yarumo (Figura 2).

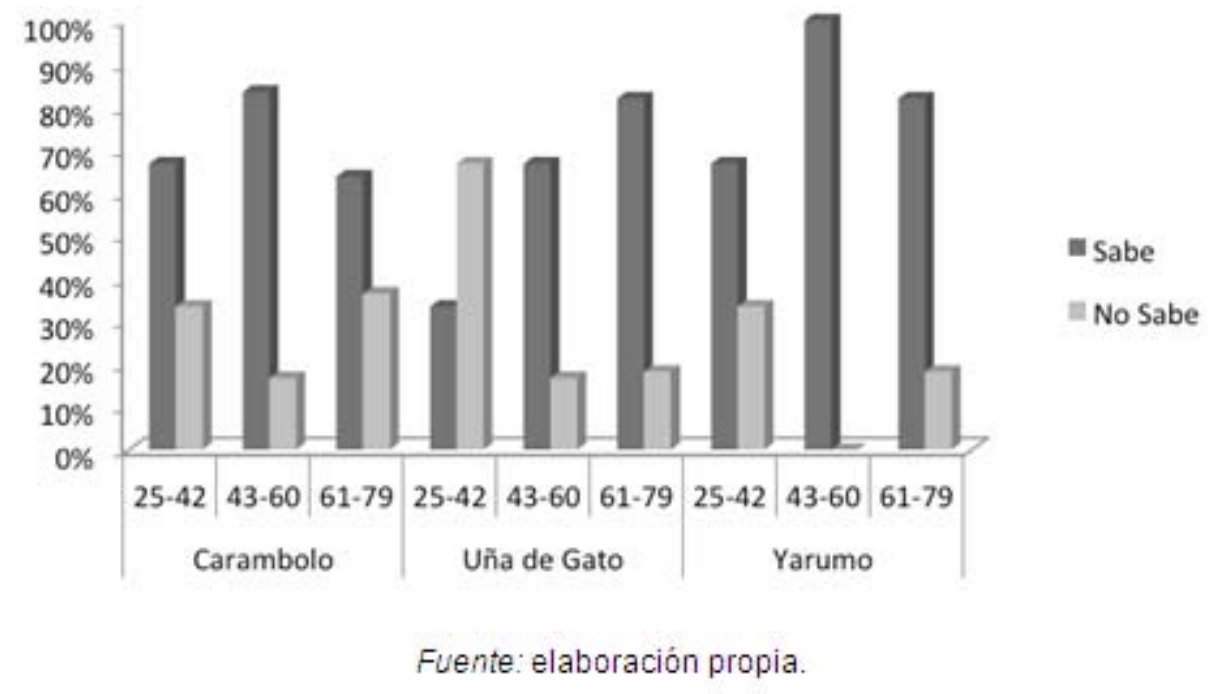

Figura 2. Conocimiento sobre el uso medicinal de las plantas analizadas por grupos etarios. 
Al indagar sobre las fuentes de conocimiento para cada una de las plantas, se encontró que la familia, especialmente los abuelos, sigue teniendo un rol significante en la transmisión del saber a través de la tradición oral y las actividades cotidianas (López, 2010). No obstante, la incidencia de la madre fue baja tanto en el Carambolo como en el Yarumo, y nula en la Uña de Gato. Es probable que el difícil acceso a esta última en el Resguardo, por escasez y las grandes distancias que hay que recorrer para obtenerla, ha hecho que se privilegie el uso otras plantas medicinales de fácil acceso (Kvist et al., 2001).

Por consiguiente, el conocimiento sobre el uso de la Uña de Gato se ha restringido más hacia los abuelos sabedores, quienes han tenido contacto con personas externas a la comunidad y de las cuales han adquirido parte de su saber. Este tipo se conoce como "conocimiento distribuido" porque no es compartido por toda la comunidad, pero sí se sabe quién la posee y se recurre a él (Zerda, 2003).

Los resultados en las tres plantas evidencian la influencia de las fuentes de información fuera del Resguardo, nacionales y extranjeras en los grupos etarios de los extremos (jóvenes y ancianos); por ende, las relaciones interétnicas que se dan con otras comunidades indígenas y con la cultura occidental inciden de forma constante en el proceso de aprendizaje tradicional dentro de Macedonia (Fajardo y Torres, 1987).

En el caso del Carambolo, se encontró que los ancianos del tercer grupo adquirieron principalmente su conocimiento de otros colombianos (Figura 3), algunos pertenecientes a comunidades indígenas de Putumayo y Caquetá que fueron al Resguardo o que conocieron fuera de él. Las personas del grupo etario de 43-60 años aprendieron en su mayoría de otros familiares como tíos o hermanos. Asimismo, los jóvenes fueron enseñados en casa a través del padre. 


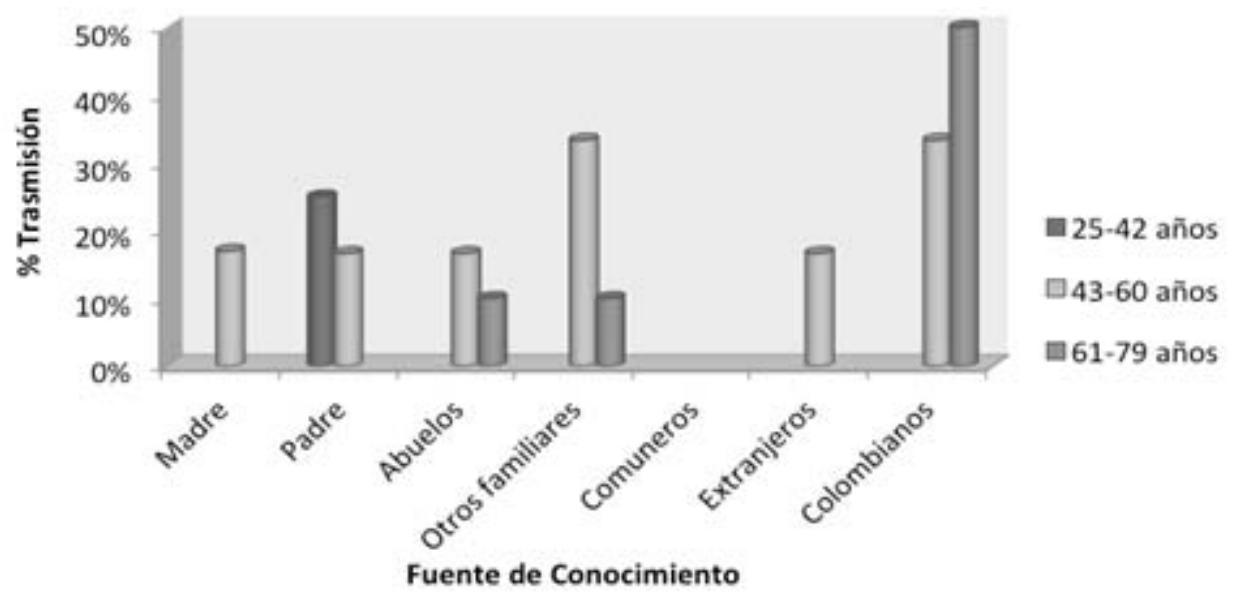

Fuente: elaboración propia.

Figura 3. Transmisión de conocimiento sobre uso medicinal del Carambolo.

De igual forma, los jóvenes aprendieron sobre los usos medicinales de la Uña de Gato a través de personas externas a la comunidad que han nacido en Colombia (Figura 4). En el segundo grupo etario se encontró una transmisión de conocimiento de la familia, especialmente de los abuelos (33\%) y en menor proporción del padre. La adquisición de saber sobre uso medicinal de esta planta en los más ancianos fue variado, siendo las fuentes más relevantes comuneros (residentes de Macedonia), personas externas de la comunidad nacionales y extranjeras, así como familiares cercanos. 


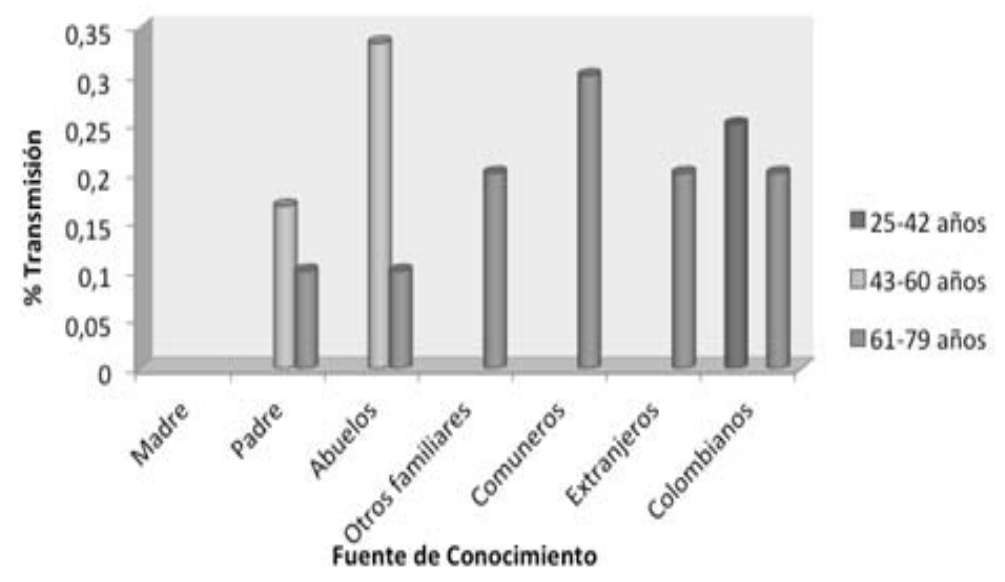

Fuente: elaboración propia.

Figura 4. Transmisión de conocimiento sobre uso medicinal de la Uña de Gato.

Todos los jóvenes entrevistados aprendieron usos medicinales del Yarumo de personas residentes de la comunidad, especialmente de los abuelos sabedores (Figura 5). Asimismo, los ancianos mayores adquirieron su conocimiento de colombianos externos a la comunidad y de otros familiares. La transmisión del uso medicinal en las personas entre $43-60$ años fue proporcional (17\%), la cual se dio por el padre, abuelos, comuneros y extranjeros.

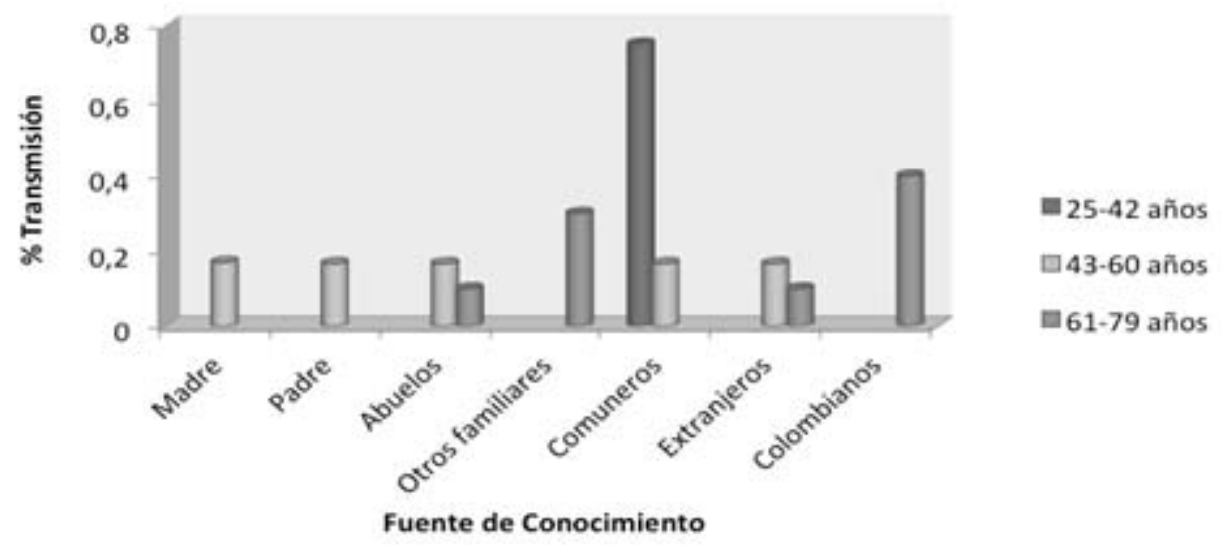

Fuente: elaboración propia.

Figura 5. Transmisión de conocimiento sobre uso medicinal del Yarumo. 


\section{Desconocimiento sobre el uso medicinal de las plantas en el Resguardo}

La disminución del modo tradicional de vida indígena en Macedonia y de su lengua, han sido punto de partida para la pérdida del conocimiento tradicional, en el que se encuentra el saber medicinal (Vengoechea, 2012). Como se observa en la Figura 6, se presentaron varias causas para el desconocimiento del uso medicinal del Carambolo, Yarumo y Uña de Gato en el Resguardo, y se destacaron la desvalorización del saber ancestral y la falta de transmisión de conocimientos por parte de los abuelos.

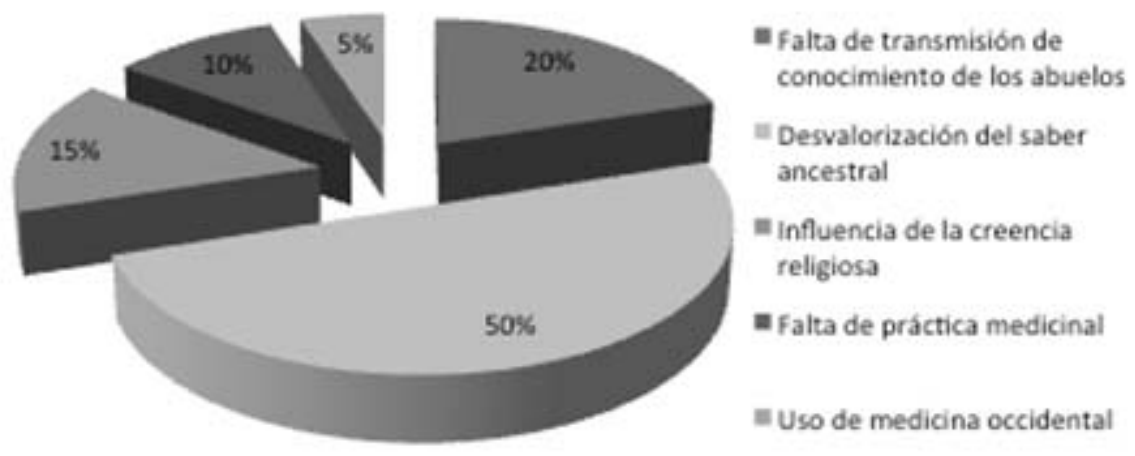

Fuente: elaboración propia.

Figura 6. Causas del desconocimiento del uso medicinal de las plantas.

En muchas comunidades indígenas la interacción con el mundo occidental y los procesos de colonización han llevado a la pérdida de la identidad cultural (Cardona, 2013). En ese sentido, hay una reducción del conocimiento ancestral que está vinculado a aspectos colectivos como el lenguaje, la espiritualidad, las relaciones sociales y la cosmovisión (Antonio, 2012).

Tal es el caso de Macedonia, donde hay personas que se identifican como mestizas pues no todos en su línea genealógica son indígenas (Barbosa, 2006). Esto ha ocasionado una reducción, sobre todo en los jóvenes, del interés de 
aprender su conocimiento tradicional como el uso de las plantas medicinales, especialmente de la Uña de Gato y el Yarumo que no han sido ampliamente utilizadas. El abuelo Alfonso Peña comentaba que no solo se está perdiendo el conocimiento tradicional sino también la lengua Tikuna:

Ya nadie le enseña su papá, ya no hablan idioma, quieren ser blancos. Se está perdiendo el idioma Tikuna por eso ellos no saben nada, qué palo es éste, cómo se llama, cómo crecieron o quién lo trajo. Ya no saben de dónde salió y ya casi no se usan plantas medicinales (com. per., 2013).

Lo anterior también evidencia la pérdida de la cadena generacional de saberes medicinales (Telles, 2013), en donde los abuelos o los padres están transmitiendo cada vez menos a sus hijos sus conocimientos, a través de sus prácticas cotidianas. Algunos le atañen esta situación al descuido de la función social de enseñanza de los abuelos (Departamento Nacional de Planeación de Colombia, 2006) y otros a la desvalorización del saber del uso de las plantas medicinales por parte de los jóvenes, a quienes no les interesa preguntar, investigar o entablar un diálogo con los mayores.

Esta última situación está sujeta al proceso de aculturización, en el cual el contacto de los jóvenes con el mundo occidental ha generado unas necesidades sociales y culturales hacia el acceso a nuevas formas de información que no son necesariamente parte de su conocimiento ancestral (Fábregas, 2012). A pesar de que haya un valor positivo dado al saber y las prácticas medicinales dentro de la comunidad, su preocupación e interés por el rescate de ese conocimiento es menor a la necesidad de integrarse a la forma de vida occidental (Ullán de la Rosa, 2000). Según Barbosa (2006, p. 90), la comunidad de Macedonia "conoce, usa y manipula [...] aquellas plantas y animales que son relevantes para garantizar su reproducción física, social y cultural".

Aunque todas las personas entrevistadas han visto o conocen el Carambolo y el Yarumo, en menor proporción la Uña de Gato, son pocos los que realmente saben su uso medicinal. Por ese motivo, la elaboración de los remedios en la comunidad se ha relegado principalmente a los abuelos sabedores y a los médicos tradicionales, que no solo conocen del uso de la planta sino que también saben su forma de preparación y la dosis en la que se debe administrar. Los demás comuneros recurren a ellos cuando necesitan tratar enfermedades más complejas o cuando no saben cuál es la enfermedad que tiene la persona. 
La creencia evangélica de la comunidad se ha apropiado de esferas sociales, políticas y culturales de Macedonia (Buitrago, 2007). Debido a esto, los rituales tradicionales son actualmente prohibidos y el chamanismo es visto como un rito pagano (Barbosa, 2006). Aunque el $77 \%$ de los indígenas entrevistados afirmaron que las iglesias del Resguardo aceptan el uso de las plantas medicinales analizadas, la creencia religiosa ha llevado a algunos a considerar que la cura a las enfermedades viene directamente de Dios y en su fe, por lo que el conocimiento medicinal no tiene la misma relevancia que en otras comunidades indígenas amazónicas (Beltrán, 2011).

De igual forma, la incidencia de la religión en sus sistemas de creencias ha generado una discrepancia en lo que se considera medicina tradicional y el chamanismo. Para ellos, el uso medicinal de las plantas solo debe centrarse en la manipulación física de las mismas sin que haya rezos o comunicación con los dueños espirituales de la plantas, pues eso lo hacen los chamanes (Barbosa, 2006) sean "brujos" buenos o malos. La necesidad de separar estas prácticas ha llevado a que los comuneros empleen otras categorías para la medicina tradicional como "medicina botánica" o "medicina casera".

La lucha interna en la comunidad entre la identidad religiosa y la identidad étnica (Beltrán, 2011) permea no solo la transmisión del conocimiento medicinal de los abuelos a las siguientes generaciones, sino también la importancia de realizar y mantener cultivos de plantas medicinales. En el relato del abuelo Israel Bastos se puede evidenciar parte de esta situación:

Yo estaba viniendo allá de la loma y él estaba sentado en el muro, cuando él me miró y como que se disgustó donde él me dice: "Ahí viene el brujo. Solamente cuando se enferma se va a donde Israel, ya no quiere orar a Dios que sane también, hay un Dios que sana también y a él ya lo tienen como Dios". Por esa razón me bajé, como que ya me hizo escuchar. Yo le pasé por el lado y no le puse cuidado. Claro que sé lo que me dijo a mí. Por esa razón casi que la iglesia no están de acuerdo con eso, solamente hay algunos, no son todos los que a veces creen... Por esa razón casi no trabajo así, dicen que a mí me tienen como un Dios. Por esa razón ya me estoy alejando de esa parte, tengo cada vez menos pacientes (com. per., 2013).

\section{Uso medicinal de las plantas de Yarumo, Uña de Gato y Carambolo}


En Macedonia se identificaron varios usos medicinales de las plantas de Carambolo, Uña de Gato y Yarumo. La mayoría de estos usos fueron reportados por los abuelos sabedores de la comunidad y otros por comuneros que residen en el Resguardo. La planta con mayor reporte de usos medicinales fue la Uña de Gato (17 usos), seguido del Carambolo (11 usos) y el Yarumo (10 usos). Adicionalmente, estos reportes fueron comparados con los encontrados en el estudio etnomedicinal de Quintana (2009) en Macedonia.

La comunidad reportó cuatro usos medicinales para el Carambolo: apendicitis, artritis, diarrea y vómito, siendo este último el punto de coincidencia entre los dos estudios (Figura 7). En la literatura también se han presentado otros usos medicinales de esta planta. Por ejemplo, Pérez y Vázquez (2004) encontraron que en la India esta planta se usa para tratar las hemorragias, las hemorroides, la fiebre y las infecciones en los ojos; mientras que en Brasil se emplea para el vómito y como diurético.

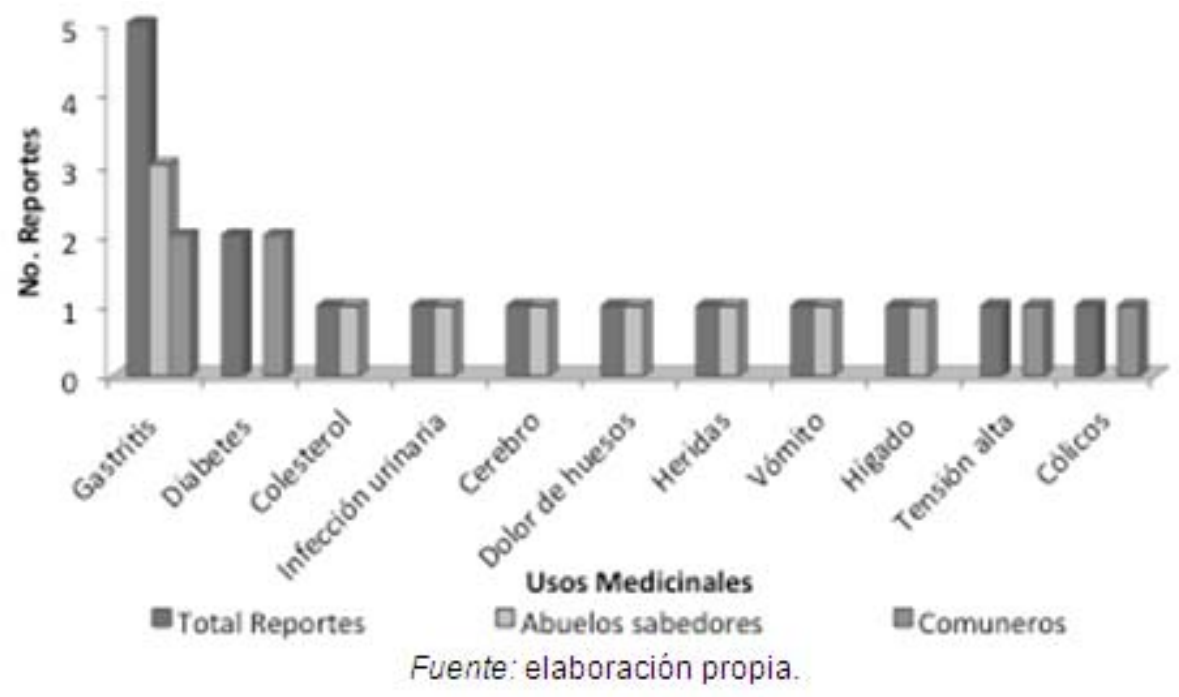

Figura 7. Usos medicinales del Carambolo reportados por la comunidad.

Recientes estudios clínicos (Das y Ahmed, 2012; Cazarolli et al., 2012; Chowdhury et al., 2013; Das et al., 2013; Wahab et al., 2013) señalan las propiedades medicinales del Carambolo como antiinflamatorio, antidiarreico, analgésico, diurético, antiemético, antihelmíntico, antifebril, antiprurítico, antibacterial y antioxidante. También se ha empleado para tratar los eczemas, la 
hipertensión y la diabetes (Almeida et al., 2011; Soncini et al., 2011; Zheng et al., 2013).

El cáncer, el reumatismo y la gastritis fueron las enfermedades que más se reportaron como tratables con la Uña de Gato en la comunidad (Figura 8). Esto coincide con los usos medicinales de esta planta encontrados en Macedonia, tales como la artritis, los cólicos, el cáncer y la inflamación (Quintana, 2009).

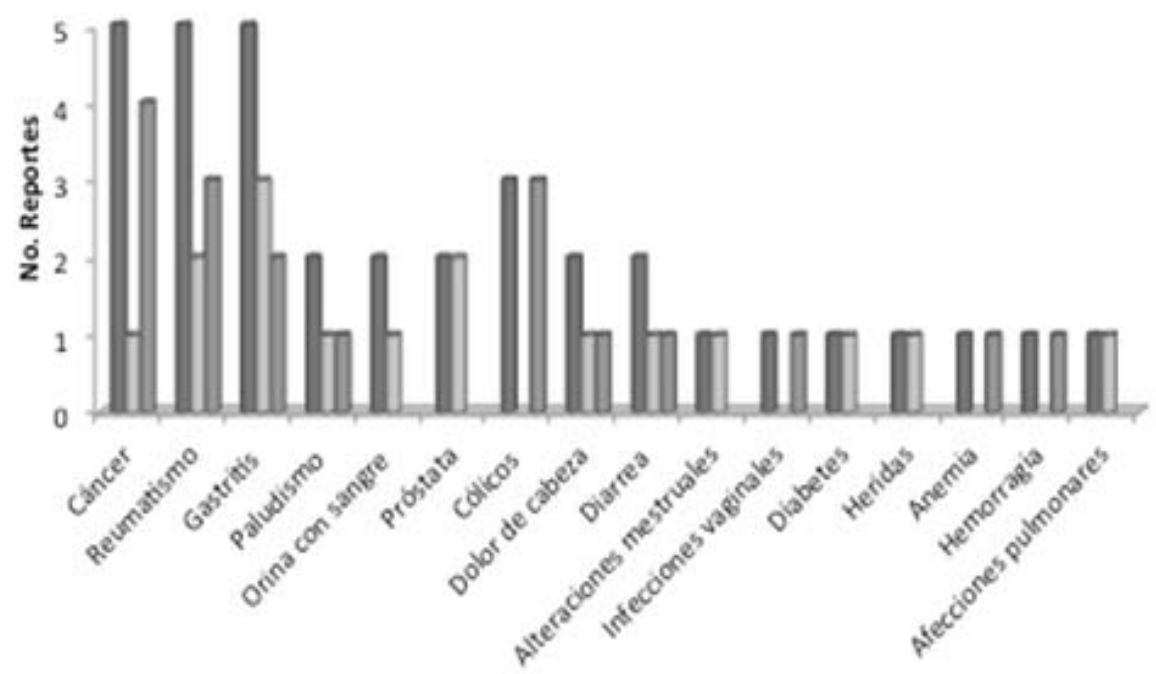

Usos medicinales

= Total Reportes $\square_{\text {Abuelos sabedores }}$ Comuneros

Fuente: elaboración propia.

Figura 8. Usos medicinales de la Uña de Gato reportados por la comunidad.

Otros reportes de usos medicinales en comunidades indígenas se han hecho en países como Colombia, Ecuador, Costa Rica, Bolivia, y principalmente Perú (Tabla 1). Estudios clínicos (Gonçalves, Dinis y Batista, 2005; Chin-Wong et al., 2008; Domingues et al., 2011; Bors et al., 2012; Santos et al., 2012, Dreifuss et al., 2013) corroboran sus efectos antiinflamatorios, inmunoestimulatorios, anticancerígenos, antioxidantes, antitumorales y antimutagénicos. De igual forma, sirve para contrarrestar enfermedades como la diabetes, la gastritis, la artritis y algunas enfermedades epidémicas como el dengue (Riva et al., 2001; Reis et al., 2008). 
Tabla 1. Reportes de usos medicinales de la Uña de Gato

\begin{tabular}{|c|c|c|}
\hline Autores & Ubicación & Usos medicinales \\
\hline Vega (2001) & $\begin{array}{l}\text { Indígenas del río } \\
\text { Apaporis, Colombia }\end{array}$ & Disentería \\
\hline $\begin{array}{c}\text { Centro de } \\
\text { Investigaciones } \\
\text { sobre Fitoterapia - } \\
\text { INFITO (2006) }\end{array}$ & $\begin{array}{l}\text { Indígenas Asháninka, } \\
\text { Amazonía peruana }\end{array}$ & $\begin{array}{c}\text { Abscesos, alergias, tumores malignos, } \\
\text { reumatismo, artritis, diabetes, cirrosis y } \\
\text { alteraciones menstruales }\end{array}$ \\
\hline Calve (2007) & Etnia Tsimané, Bolivia & Dolor de estómago, diarrea \\
\hline Molina (2007) & $\begin{array}{l}\text { Comunidad de } \\
\text { Tambopata, Madre de } \\
\text { Dios, Perú }\end{array}$ & $\begin{array}{l}\text { Reumatismo, úlcera, prostatitis, artritis, } \\
\text { infecciones urinarias, disentería }\end{array}$ \\
\hline Rengifo (2007) & $\begin{array}{l}\text { Comunidad Nativa Bora } \\
\text { de Brillo Nuevo, } \\
\text { Amazonía peruana }\end{array}$ & $\begin{array}{l}\text { Artritis, cáncer, reumatismo, } \\
\text { sarampión }\end{array}$ \\
\hline Venutolo (2010) & $\begin{array}{c}\text { Comunidades indígenas } \\
\text { de Costa Rica }\end{array}$ & $\begin{array}{l}\text { Gastritis, artritis, cáncer, VIH y fortificante } \\
\text { del sistema inmunológico }\end{array}$ \\
\hline $\begin{array}{c}\text { Trujillo y González } \\
\text { (2011) }\end{array}$ & $\begin{array}{c}\text { Comunidad Coreguaje, } \\
\text { Putumayo }\end{array}$ & Inflamaciones, antioxidante \\
\hline Heras (2012) & $\begin{array}{l}\text { Comunidad de } \\
\text { Parroquia Tarqui, } \\
\text { Ecuador }\end{array}$ & Infecciones urinarias \\
\hline
\end{tabular}

Fuente: elaboración propia.

Sumado a lo anterior, en el Resguardo se reportó el uso popular del Yarumo para la diabetes, el SIDA y como relajante (Quintana, 2009). En la Figura 9 se observa que las principales enfermedades que los indígenas de esta comunidad pueden tratar con esta planta son el colesterol, las afecciones pulmonares y la tensión alta. 


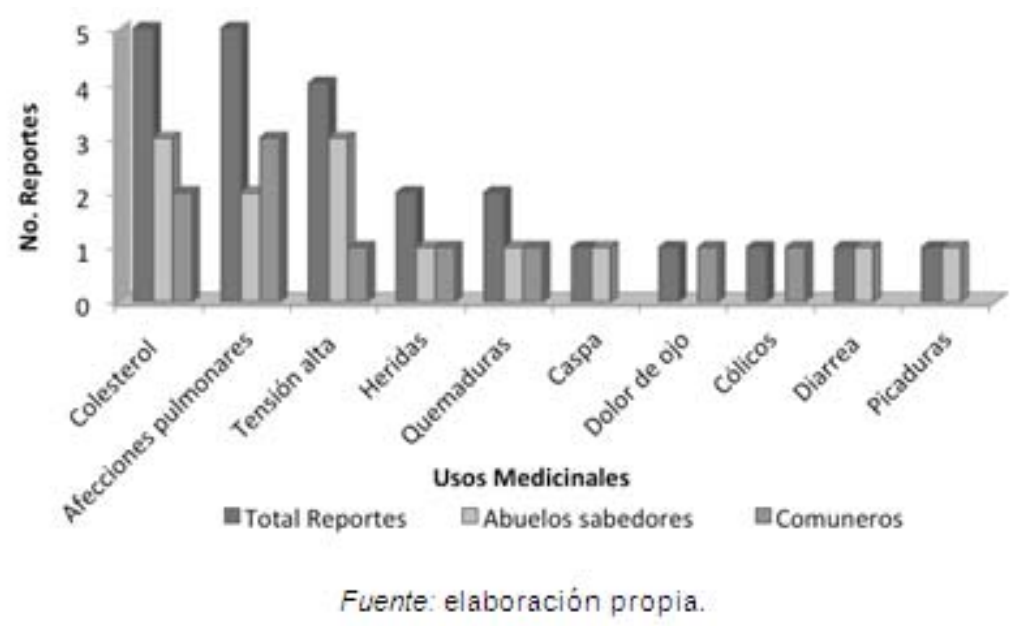

Figura 9. Usos medicinales del Yarumo reportados por la comunidad.

Vega (2001) encontró que los Indígenas del río Apaporis de Colombia usan el Yarumo como diurético, antidiarreico, estimulante del corazón y para tratar la blenorragia; mientras que De Oliveira et al. (2012) indicaron que los indígenas Quilombolas de la Amazonía brasileña lo emplean parar curar la erisipela (infección cutánea). Asimismo, Molina (2007) señaló que esta planta se usa para las picaduras de insectos y las hemorragias en la Comunidad de Tambopata, Madre de Dios, Perú. Aún no hay estudios sobre las propiedades medicinales del Yarumo; sin embargo, se conoce que la mezcla de esta planta con la Coca (Erythroxylumsp.) conocida como Mambe sirve para reducir la acidez (Martins-da Silva et al., 2012).

\section{Prácticas medicinales en la preparación de remedios}

Las formas tradicionales de preparación de los remedios en la comunidad son infusiones o zumos, chapeo, decocción y raspado. Asimismo, la mayoría de los tratamientos son administrados de forma oral, tópica y en baños. En muchos casos los preparados se entierran o se dejan en el sereno toda la noche, ya que consideran que este paso potencia el efecto del remedio. Por ejemplo, en la comunidad se realiza este proceso para tratar las infecciones urinarias con Carambolo y la gastritis con Uña de Gato.

El fruto del Carambolo se licua sin dulce y se toma tres veces al día por nueve días para tratar el colesterol y la tensión alta; mientras que para la gastritis se puede tomar solo o se mezcla con naranjas por un periodo de 30 días. El médico 
tradicional Israel Bastos comentó que al mezclar el carambolo rallado con leche y huevos se tiene una bebida que sirve como reconstituyente para el cerebro.

Para la preparación de los remedios a base de Uña de Gato, se emplea principalmente la corteza, la cual se raspa, se chapea y se pone a hervir para tratar el cáncer, la diabetes, las alteraciones menstruales, la hematuria, la diarrea y el paludismo. Cuando se corta este bejuco sale agua que utilizan para tratar la gastritis y el reumatismo.

Algunos abuelos sabedores señalaron que esta corteza se mezcla con bebidas fermentadas como el aguardiente para dar energía y tratar el dolor de huesos. También, se puede combinar con otras plantas como la flor de algodón parar la gastritis o con miel de abejas para las afecciones pulmonares. La toma de estos remedios se restringe a intervalos de 15 días o menos, ya que consideran que la ingesta excesiva de preparados con esta planta puede ser tóxica para el cuerpo.

Las partes que más emplean para hacer remedios con Yarumo son el cogollo, las hojas, la corteza y la raíz. Es posible que el uso de estas partes vegetativas sea mayor que los frutos (en el caso del Carambolo), ya que éstas se encuentran disponibles a lo largo del año (Trujillo y González, 2011).

Con la sabia que se encuentra en el interior del cogollo (sola o con gotas de limón) se tratan las afecciones pulmonares, la cual se administra por cucharadas tanto a niños como a adultos. También, se calienta el cogollo y el jugo que suelta se coloca como cataplasma para bajar los hinchazones o se chapea con agua tibia para tratar la caspa, lavando el cabello diariamente por 15 días.

La comunidad utiliza en los preparados las hojas secas que se han caído del árbol. Entre 9 y 10 hojas se hierven con agua y se toma por un periodo de tiempo que va desde los tres días hasta los 15 días, para el colesterol. En el caso del tratamiento de los cólicos, la hoja se calienta y se pone sobre el estómago; mientras que para tratar la tensión alta se pueden emplear tanto las hojas como la corteza, la cual se corta en trozos pequeños y se cocina.

Asimismo, la hoja se quema hasta que queda una ceniza que se coloca sobre la piel para calmar la rasquiña de las picaduras o el ardor de las quemaduras. La raíz se emplea para el dolor de ojos, ya que se lava y se le hace punta para obtener el líquido que tiene en su interior. Esta agua se coloca directamente sobre los ojos y se espera 15 minutos a que haga efecto. 
Estas formas de preparación de los remedios están sujetas a una dosis que no es fija, sino que se emplea hasta que se ve la mejoría en el individuo. La aproximación de la cantidad en cuanto a la preparación como a la dosis de un remedio es difícil de establecer, ya que los comuneros emplean formas de medición como "tozas", "cucharadas", "toma", "número de hojas".

La dieta en algunas comunidades indígenas se considera que tiene una influencia sobre el tratamiento de una enfermedad (Dos Santos y Ferreira, 2002), por lo que debe ser considerada a la hora de utilizar los remedios (De Oliveira et al., 2012). En el caso de Macedonia, el indígena Cocama Pablo Pérez comentó que cuando se está tomando el agua con hoja de Yarumo se debe evitar el café, el dulce y las grasas: "Eso sí, la dieta es importante. Si usted no hace dieta mejor no tome. Café no debe tomar, dulce un poquito, no puedes comer aceite durante un mes" (com. per., 2013).

\section{CONCLUSIONES}

La adquisición del conocimiento medicinal sobre las plantas de Carambolo, Uña de Gato y Yarumo ha sido permeada por procesos migratorios, especialmente por el transnacionalismo. Esto se debe a que los abuelos que llegaron a la comunidad durante su conformación, trajeron consigo conocimientos de comunidades amazónicas ribereñas y del Putumayo, así como de indígenas peruanos y brasileños. De igual forma, el constante flujo de personas por la zona fronteriza ha llevado a que aún se mantenga ese intercambio de saberes dentro y fuera del Resguardo.

Los usos medicinales de estas plantas son conocidos en su mayoría por los abuelos sabedores, quienes tienen mayor experiencia con su manejo y preparación. Sin embargo, la dificultad de encontrar la Uña de Gato en medio natural y el poco uso, tanto del Yarumo como del Carambolo, han llevado a que se privilegie el uso medicinal de otras plantas, y por ende, se relegue la preparación de estos remedios. La familia, en especial el padre y los abuelos, sigue siendo la principal fuente de transmisión del conocimiento medicinal dentro de la comunidad, por medio de la tradición oral y las actividades cotidianas. Otras fuentes correspondieron a personas externas a la comunidad de origen colombiano y 
extranjeras, que influyeron principalmente en el aprendizaje de individuos mayores de 42 años.

No obstante, la apertura de fronteras (físicas y comerciales) sumado al intercambio cultural y de costumbres con el mundo occidental, ha conducido a la comunidad de Macedonia hacia un fenómeno de de-territorialización y de-localización de la cultura (Colajanni, 2005). En este sentido, los elementos culturales de los indígenas del Amazonas se han mezclado fuera de su contexto de origen y siguen perdiendo su carácter tradicional. Esta situación se ve reflejada en la desvalorización del saber medicinal, que lleva a un desconocimiento sobre las propiedades y usos medicinales de las plantas de Carambolo, Uña de Gato y Yarumo.

Los preceptos arraigados de la creencia evangélica, han contribuido a volver obsoletas las formas alterativas de ideas o visiones que no son aceptables en la concepción moderna de conocimiento etnomedicinal dentro del Resguardo. En consecuencia, la medicina tradicional adquirió otras connotaciones de medicina botánica o medicina casera, en la que se le despoja de su valor cultural, su carácter espiritual y se enfoca en un uso terapéutico o curativo. Esta problemática conduce a una pérdida acelerada de la transmisión intergeneracional del saber medicinal, lo que se traduce en un descuido de la función social de enseñanza de los abuelos. Por consiguiente, se configura un conocimiento distribuido (dado a los abuelos sabedores) y se genera la falta de interés en el conocimiento medicinal, especialmente en la población joven.

Sumado a lo anterior, la comunidad hizo el reconocimiento de algunas de las propiedades medicinales de las plantas de esta investigación, en especial de la Uña de Gato que tuvo el mayor registro de usos curativos. La comparación de los reportes hechos anteriormente dentro de la misma comunidad, junto con otros realizados en otras comunidades amazónicas, ha permitido la validación de dicha información. Muchos de los usos reportados por los indígenas estaban ligados a un proceso basado en las experiencias y observaciones, que con el tiempo ha llevado a una confianza generalizada en sus propiedades terapéuticas y en las formas de preparación y dosificación de los remedios.

Es importante resaltar que la medicina tradicional indígena es un conjunto de conocimientos, prácticas y creencias de una cultura, que le dan un sentido holístico al concepto de salud bajo un dominio físico, espiritual y ambiental (Cardona y Rivera, 2012). No obstante, hay una fragmentación de este concepto, pues la 
comunidad enfoca el poder curativo de las plantas de Carambolo, Yarumo y Uña de Gato en el tratamiento de aspectos físicos del paciente, por lo que el tratamiento espiritual queda en manos de la fe evangélica.

\section{REFERENCIAS}

- Alcaldía de Leticia. (2002). Plan básico de Ordenamiento Territorial. Documentoparticipación urbana y rural. Leticia: Alcaldía Municipal de Leticia.

- $\quad$ Almeida Cabrini, D.; Hunger, H.; Imazu, P.; Delai, C.; Fernandes, D.A., et al. (2011). Analysis of the Potential Topical Anti-Inflammatory Activity of Averrhoa carambola L. in Mice. Evidence-Based Complementary and Alternative Medicine, Hindawi Publishing Corporation.

- Antonio Miguel, N.B. (2012). Saberes con rostros de mujeres indígenas, conocimientos tradicionales y actividades agrícolas productivas, en el Ejido el Remolino, municipio de Huitiupán, Chiapas. Tesis de grado en Licenciatura en Desarrollo Sustentable. Universidad Intercultural de Chiapas, Chipas.

- Barbosa, C.E. (2006). El desarrollo propio en Macedonia: Una mirada al desarrollo indígena en la ribera amazónica colombiana. Tesis de Maestría en Estudios Amazónicos. Universidad Nacional de Colombia-Sede Amazonía, Leticia.

- Beltrán, W.M. (2011). Impacto social de la expansión de los nuevos movimientos religiosos entre los indígenas colombianos. Revista Colombiana de Sociología, 36(1), 35-54.

- Bors, M.; Michałowicz, J.; Pilarski, R.; Sicińska, P.; Gulewicz, K. y Bukowska, B. (2012).Studies of biological properties of Uncaria tomentosa extracts on human blood mononuclear cells. Journal of Ethnopharmacology, 142(3), 669-678.

- Buitrago, A.I. (2007). Trayectorias vitales, memoria familiar y memoria histórica en Macedonia, una comunidad indígena del Trapecio 
Amazónico colombiano. Tesis de Maestría en Estudios Amazónicos. Universidad Nacional de Colombia-Sede Amazonía, Leticia.

- Calvet, L. (2007). La división entre el conocimiento medicinal local y la medicina occidental. Caso de estudio entre los Tsimane' en la Amazonía Boliviana. Perifèria, 7, 1-28.

- Cardona, J.A. (2013). Vínculo entre mestizaje y salud en un sistema médico de una comunidad indígena Colombiana. Revista Cubana de Salud Pública, 39(4), 651-664.

- Cardona, J.A. y Rivera, Y. (2012). Representaciones sociales sobre medicina tradicional y enfermedades foráneas en indígenas Embera Chamí de Colombia. Revista Cubana de Salud Pública, 38(3), 471-483.

- Cazarolli, L.H.; Demarchi, V.; Fontana, D.; Hunger, H.; Costa, I.M.; Geraldo, M., et al. (2012). Anti-hyperglycemic action of apigenin-6-C- $\beta$-fucopyranoside from Averrhoa carambola. Fitoterapia, 83(7), 1176-1183.

- Centro de Investigaciones sobre Fitoterapia - INFITO. (2006). Plantas medicinales para enfermedades reumáticas. Madrid: Editorial Complutense UCM.

- Chin-Wong, J.L.; Vásquez, P.O.; Monge, A. y Moreno, M. (2008). Efecto de Uncaria tomentosa en la mutagénesis de Salmonella typhimurium inducida por 7,12 dimetilbenzantranceno con activación metabólica in vitro. Revista Cubana de Plantas Medicinales, 13(2). Recuperado de http://scielo.sld.cu/scielo.php?pid=S102847962008000200003\&script=sci_arttext

- Chowdhury, A.N.; Ashrafuzzaman, M.; Ali, H.; Lutfun, L. y Zinnah, M.A. (2013).Antimicrobial Activity of Some Medicinal Plants against Multi Drug Resistant Human Pathogens. Advances in Bioscience and Bioengineering, 1(1), 1-24.

- Colajanni, A. (2005). Viejas y nuevas identidades indígenas en el cuadro de los procesos de globalización en la Amèrica Latina de hoy. En Kowii, A. (Ed.), Identidad linguística de los pueblos indígenas de la región andina. Quito: Ediciones Abya-Yala. 
- $\quad$ Das, J.; Zulon, D.; Ayan, S.; Suza, M.; Prosenjit, B.; Mominur, R., et al. (2013). A comprehensive study on antioxidant, antibacterial, cytotoxic and phytochemical properties of Averrhoa carambola. International Journal of Bioassays, 2(5),803-807.

- Das, N.B. y Ahmed, M. (2012). Analgesic activity of the fruit extract of Averrhoa carambola. International Journal of Life Science Biotechnology and Pharma Research, 1(3), 22-26.

- De Oliveira, D.R.; Guimarães, G.; Goncalves, N.; Neves, M. y Guimarães, S. (2012).Ethnomedical Knowledge. Among the "Quilombolas" from the Amazon region of Brazil qith a special focus on plantas used as nervous system tonic. En Mahendra, R.; Cordell, G.A.; Martínez, J.L.; Marinoff, M. y Rastrelli, L. (Eds.), Medicinal Plants: Biodiversity and Drugs. Boca Ratón, FL: Taylor \& Francis Group.

- Departamento Nacional de Planeación de Colombia. (2006). Los pueblos indígenas de Colombia en el umbral del nuevo milenio. Bogotá: Alto Comisionado de las Naciones Unidas para los Refugiados - ANCUR.

- Dias de Avila-Pires, F. (2007). Indigenous Knowledge and Sustainable Development in Brazil. En Boon, E.K. y Hens, L. (Eds.), Indigenous knowledge systems and sustainable development: relevance for Africa. USA: Kamla-Raj Enterprises.

- Domingues, A.; Sartori, A.; Golim, M.; Marino, L.; Camargo, L.; Lumi, L., et al. (2011). Prevention of experimental diabetes by Uncaria tomentosa extract: Th2 polarization, regulatory $T$ cell preservation or both? Journal of Ethnopharmacology, 137(1), 635-642.

- Dos Santos, F.S. y Ferreira Muaze, M. (2002). Tradições em Movimento: uma etnohistória da saúde e da doença nos vales dos rios Acre e Purus. Brasilia: Paralelo 15.

- $\quad$ Dreifuss, A.A.; Bastos-Pereira, A.L.; Fabossi, I.; Lıvero, F.; Stolf, A.M.; Alves, C.E., et al.(2013). Uncaria tomentosa Exerts Extensive Anti-Neoplastic Effects against the Walker-256 Tumour by Modulating Oxidative Stress and 
Not by Alkaloid Activity. Plos One, 8(2). Recuperado de http://www.ncbi.nlm.nih.gov/pmc/articles/PMC3567083

- Fábregas, A. (2012). De la teoría de la aculturacíon a la teoría de la interculturalidad. Educación y asimilación: el caso mexicano. Intercultural Communication Studies XXI, 1, 1-8.

- Fajardo, G. y Torres, W. (1987). Ticuna. En Instituto Colombiano de Antropología,Introducción a la Colombia Amerindia. Bogotá: Ministerio de Educación Nacional.

- Gonçalves, C.; Dinis T. y Batista, M.T. (2005). Antioxidant properties of proanthocyanidins of Uncaria tomentosa bark decoction: a mechanism for anti-inflammatory activity.Phytochemistry, 66(1), 89-98.

- Goulard, J.P. (2003). Cruce de identidades. El Trapecio Amazónico colombiano. En García, C.I. (Ed.), Fronteras, metáforas y territorios. Medellín: INER - Universidad de Antioquia.

- Heras, M.F. (2012). Estudio etnobotánico en las explotaciones agropecuarias de la Parroquia Tarqui. Tesis de grado en Ingeniería Ambiental. Universidad Estatal Amazónica, Puyo, Ecuador.

- Kvist, L.P.; Oré, I.; Gonzales, A., y Llapapasca, C. (2001). Estudio de plantas medicinales en la Amazonía peruana: una evaluación de ocho métodos etnobotánicos. Folia Amazónica, 2(1), 53-72.

- López Urrego, A.P. (2010). Una noción de territorio y los sistemas de información geográfica participativos: Experiencia en una comunidad indígena del Amazonas colombiano. Revista UD y Geomática, 4(1), 3-14.

- Luque, J.C. (2012). Notas sobre migración y ciudadanía: una crítica sur-norte a los enfoques transnacionales funcionalistas. Tukuy Migra, 3(1), 2-23.

- Martins-da-Silva, R.C.; Gaglioti, A.L.; Tavares, L.; Gomes, J.I. y Margalho, L. (2012). Conhecendo Espécies de Plantas da Amazônia: Imbaubão (Cecropia sciadophylla Mart. - Urticaceae). Comunicado Técnico 234. Embrapa, Belem. 
- Molina, Y. (2007). Estudio etnobotánico y etnofarmacológico de plantas medicinales de Tambopata, Madre de Dios, Perú. Ciencia y Desarrollo, 14(3), 7-26.

- Organización Panamericana de la Salud. (2006). Medicina indígena tradicional y medicina convencional. San José de Costa Ric: Autor.

- Oyuela, A. y Vieco, J. (1999). Mitades, clanes y casas del Trapecio Amazónico colombiano: una perspectiva numérica de los Tikuna. Amazonia em Cuadernos, 5, 39-67.

- Page, J.T. (1995). Health Policy and Legislation Concerning Traditional Indigenous Medicine in Mexico. Cadernos de Saúde Púbica, 11(2), 201-211.

- Parques Nacionales Naturales. (2005). Plan de Manejo del Parque Nacional Natural Amacayacu. Leticia: Autor.

- Peña-Venegas, C.P.; Mazorra, A.; Acosta, L.E. y Pérez, M.N. (2009). Seguridad alimentaria en comunidades indígenas del Amazonas: ayer y hoy. Bogotá: Instituto Amazónico de Investigaciones Científicas Sinchi.

- Pérez, M.H., y Vázquez, V. (2004). Carambolo (Averroha carambola L.) su cultivo y produccion en Nayarit. Instituto Nacional de Investigaciones Forestales, Agrícolas y Pecuarias. Santiago Ixcuintla, Nayarit, México: Centro de Investigación Regional del Pacífico Centro.

- Pezo, R. (2006). Diversidad Cultural en la Amazonia Peruana. Honolulu, Hawai: Atlantic International University.

- Pinzón, H. y Suárez, R. (1992). Las mujeres lechuza, historia, cuerpo y brujería en Boyacá. Serie Amerindia No. 4. Bogotá: CEREC, Instituto Colombiano de Antropología.

- Quintana, R.F. (2009). El verdadero guardián del oro verde, estudio etnobotánico en la comunidad Tikuna del Alto Amazonas, Macedonia. Tesis de grado en Licenciatura en Biología. Universidad Distrital Francisco José de Caldas, Bogotá. 
- $\quad$ Reis, S.R.; Valente, L.M.; Sampaio, A.L.; Siani, A.C.; Gandini, M.; Azeredo, E.L., et al.(2008). Immunomodulating and antiviral activities of Uncaria tomentosa on human monocytes infected with Dengue Virus-2. International Immunopharmacology, 8(3), 468-476.

- Rengifo, E. (2007). Iquitos: Instituto de Investigaciones de la Amazonía Peruana (IIAP).

- Riva, L.; Coradini, D.; Di Fronzo, G.; De Feo, V.; De Tommasi, N.; De Simone, F. y Pizza, C. (2001). The antiproliferative effects of Uncaria tomentosa extracts and fractions on the growth of breast cancer cell line. Anticancer Research, 21(4), 2457-2461.

- Sánchez, M.A. (1999). Historia, teoría y método de la medicina: introducción al pensamiento médico. Barcelona: Mansón.

- $\quad$ Santos, M.C.; Farias, I.L.; Gutierres, J.; Dalmora, S.L.; Flores, N.; Farias, J., et al. (2012).Uncaria tomentosa-Adjuvant Treatment for Breast Cancer: Clinical Trial. Evidence-Based Complementary and Alternative Medicine, 1, 1-8.

- Soncini, R.; Santiago, M.B.; Orlandi, L.; De Olivera, G.; De Morales, I., Peloso, A.L., et al.(2011). Hypotensive effect of aqueous extract of Averrhoa carambola L. (Oxalidaceae) in rats: An in vivo and in vitro approach. Journal of Ethnopharmacology, 133(2), 353-357.

- Telles, L. (2013). Conocimiento botánico tradicional en jóvenes del área rural maya, estudiantes de biología en el Instituto Tecnológico de Conkal en Yucatán. XII Congreso Nacional de Investigación Educativa. Guanajuato, México. Consejo Mexicano de Investigación Educativa. Universidad de Guanajuato.

- Tello, O.; García, R. y Vásquez, O. (2002). Conservación de Averrhoa carambola"carambola" por azúcar y calor. Revista Amazónica de Investigación Alimentaria, 2(1), 49-58.

- Tobón, M. y Ochoa, G. (2010). De vacaciones en la Amazonia, Turismo y nuevas formas de trabajo en poblaciones indígenas. En Tobon, M. y Duque, S.R. (Eds.), Remando a varias manos. Investigaciones desde la 
Amazonía (pp. 39-62). Leticia: Universidad Nacional de Colombia, Sede Amazonia - Instituto Amazónico de Investigaciones.

- Trujillo, W. y González, V.H. (2011). Plantas medicinales utilizadas por tres comunidades indígenas en el noroccidente de la Amazonia colombiana. Mundo Amazónico, 2, 283-305.

- Ullán de la Rosa, F.J. (2000). Los indios ticuna del Alto Amazonas ante los procesos actuales. Revista Española de Antropología Americana, 30, 291336.

- Unigarro, D.E. (2011). Alegría sin fronteras entre Brasil, Colombia y Perú: de cómo "tres países hermanos" celebran el nacionalismo. Maguaré, 26(1), 263297.

- Vargas, C. (2010). Del yagé al mito de Gútapa: mirada retrospectiva. Cuadernos de Literatura, 14(27), 156-169.

- Vega, M. (2001). Etnobotánica de la Amazonia peruana. Quito: Ediciones Abya-Yala.

- Vengoechea, C. (2012). El conocimiento tradicional: Retos y perspectivas. Revista Colombia Amazónica, 5, 39-42.

- Venutolo, S.A. (2010). Establecimiento in vitro y cultivo de células de la uña de Gato (Uncaria tomentosa) (Willd.) D.C. Tecnología en Marcha, 23(5), 24-33.

- Wahab, S.; Hussain, A.; Farooqui, A.H. y Ahmad, P. (2013). Authentication and quality evaluation of an important ayurvedic drug Averrhoa carambola linn leaves. Asian Journal od Pharmaceutical and Clinical Research, 6(4), 52-56.

- Zerda, A. (2003). Propiedad intelectual sobre el conocimiento vernáculo. Bogotá: Facultad de Ciencias Económicas, Universidad Nacional de Colombia.

- Zheng, N.; Lin, X.; Wen, Q.; Zhang, S.; Huang, J.; Xu, X., et al. (2013). Effect of 2-dodecyl-6-methoxycyclohexa-2,5-diene-1,4-dione, isolated from Averrhoa carambola L. (Oxalidaceae) roots, on advanced glycation end-product- 
mediated renal injury in type 2 diabetic KKAy mice. Toxicology Letters, 219(1), 77-84.

- Zuluaga, G. (1999). Elementos de reflexión para la conservación de la diversidad biológica y cultural. Taller sobre experiencias prácticas en gestión de Áreas Protegidas por lo pueblos indígenas de Iberoamérica. Cartagena de Indias: Ministerio de Medio Ambiente.

- Zuluaga, G. y Correa, C. (2002). Medicinas tradicionales: Introducción al estudio de los sistemas tradicionales de salud y su relación con la medicina moderna. Bogotá: Kimpres.

1. Este artículo contiene parte del trabajo publicado en el libro Sistemas productivos sostenibles de plantas medicinales en el Amazonas, por la Editorial Académica Española. ISBN 3659044547.

2. Ms. en Desarrollo Sustentable y Gestión Ambiental. Universidad Distrital Francisco José de Caldas. Bogotá, Colombia. lina.garzong@hotmail.com. ORCID: 0000-0001-8035-8755

3. En este Resguardo Indígena se pueden encontrar varios grupos étnicos, donde la mayoría de la población pertenece a la etnia Tikuna $(63,3 \%)$ y a otros siete grupos étnicos: Cocamas, Yaguas, Mirañas, Huitotos, Tanimucas, Boras y Mestizos (Oyuela y Vieco, 1999; Peña-Venegas et al., 2009).

Para citar este artículo: Garzón-Garzón, L.P. (2016). Conocimiento tradicional sobre las plantas medicinales de Yarumo (Cecropia sciadophylla), Carambolo (Averrhoa carambola) y Uña de Gato (Uncaria tomentosa) en el Resguardo Indígena de Macedonia, Amazonas.Revista Luna Azul, 43, 386-414. Recuperado de

http://200.21.104.25/lunazul/index.php?option=com_content\&view=article\&id=206

Esta obra está bajo una Licencia de Creative Commons Reconocimiento CC BY

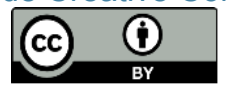

